\title{
Integrating medical abortion into safe abortion services: experience from three pilot sites in South Africa
}

\author{
Mary Kawonga, Kelly Blanchard, Diane Cooper, Lee Cullingworth, Kim Dickson, Teresa Harrison, \\ Claire von Mollendorf, Beverly Winikoff
}

\begin{abstract}
Background and methodology South Africa's Choice on Termination of Pregnancy Act of 1996 provides for safe termination of pregnancy (TOP) in designated facilities in the public and private health sectors. In 2001, mifepristone-misoprostol medical abortion was approved for TOP up to 56 days, but this method is not yet available in the public sector. Information on the operational requirements for integrating mifepristone-misoprostol medical abortion into South Africa's public sector safe abortion services is required to guide policy decisions. This study trained health workers to provide medical abortion to 290 women attending three TOP sites. Prospective data were collected to ascertain women's experience of the method, pregnancy outcome, women's and provider's acceptability of the method, and the operational requirements for providing medical abortion.
\end{abstract}

Results Twenty-nine (10\%) women were lost to follow-up; $261(90 \%)$ women had a confirmed abortion outcome, of whom $93 \%$ had a complete abortion. Given the option, the vast majority of women opted to use misoprostol at home. No serious side effects were reported; pain (66\%), and heavy bleeding $(67 \%)$ were the most common side effects. Most $(96 \%)$ women were very satisfied with the experience. Health providers were satisfied with providing medical abortion and recommended its introduction to complement existing surgical TOP services.

Discussion and conclusion This study demonstrates that integration of medical abortion into public sector services is feasible. The results of this study will guide policy decisions about integrating medical abortion into South Africa's public sector safe abortion services, within the context of the existing enabling legislative framework.

Keywords medical abortion, mifepristone, misoprostol, public sector safe abortion services, South Africa

J Fam Plann Reprod Health Care 2008; 34(3): 159-164 (Accepted 12 December 2007)

\section{Introduction}

Numerous clinical studies in the literature report the efficacy of mifepristone-misoprostol medical abortion in both developed and developing countries. ${ }^{1-6}$ This regimen, with an efficacy of 92-98\% for early termination of pregnancy (TOP), is effective outside clinical trial settings, ${ }^{7-9}$ but is not registered and available in many countries where abortion is legal. The complexity of the original mifepristone-misoprostol regimen, requiring $600 \mathrm{mg}$ mifepristone for pregnancies up to 49 days and three health facility visits, may limit its implementation in less developed countries. ${ }^{10}$ Alternative regimens including $200 \mathrm{mg}$ mifepristone and self-administration of misoprostol at home are simpler and have a comparable

School of Public Health, University of the Witwatersrand, Johannesburg, South Africa

Mary Kawonga, MBChB, FCPHM (SA), Senior Specialist

Ibis Reproductive Health, Cambridge, MA, USA

Kelly Blanchard, SM, Senior Researcher

Women's Health Research Unit, University of Cape Town, Cape Town, South Africa

Diane Cooper, PhD, Director

Lee Cullingworth, MBChB, DFSRH, Senior Researcher

Constella Group, Durham, NC, USA

Teresa Harrison, SM, Program Advisor

Reproductive Health and HIV Research Unit, Johannesburg, South Africa

Kim Dickson, MBChB, MSc, Clinical Director

Claire von Mollendorf, MBBCh, BScMedSc, Deputy Director

Gynuity Health Projects, New York, NY, USA

Beverly Winikoff, MD, MPH, Director

Correspondence to: Dr Mary Kawonga, School of Public Health, University of the Witwatersrand, 7 York Road, Parktown, Johannesburg, Gauteng 2193, South Africa.

E-mail: mary.kawonga@wits.ac.za

\section{Key message points}

- This operations research study shows that it is feasible to integrate medical abortion into the South African public health care sector. Medical abortion was acceptable to women and health care providers in this pilot study.

- A two-visit medical abortion regimen incorporating home use of misoprostol was implemented in this study and was acceptable to women who used it. Mechanisms for feasibly integrating this option into South African public sector termination of pregnancy services should be explored.

- A high medical abortion success rate comparable to international experiences for early pregnancies up to 56 days was achieved in this pilot study, without compromising patient safety.

efficacy. ${ }^{4,11}$ Home use of misoprostol is safe, acceptable and feasible even in less developed contexts, ${ }^{12-14}$ and a modified regimen extending the limit to 56 days achieves success rates of $92-97 \% .10,13$

\section{Medical abortion in South Africa}

South Africa's Choice on Termination of Pregnancy Act of 1996 allows TOP on request up to 12 weeks from the last menstrual period, provided by a certified nurse practitioner or doctor. ${ }^{15}$ Manual vacuum aspiration (MVA) is offered free of charge through designated public sector clinics and hospitals to women requesting terminations for pregnancies up to 12 weeks. The Termination of Pregnancy Implementation Guidelines of 1997 made provision for the inclusion of medical abortion into health services if it became available; 16 and in 2001, the South Africa Medicines Control Council (MCC) approved the use of mifepristone with misoprostol for TOP up to 56 days (8 weeks) from the last menstrual period (LMP). This regimen includes $600 \mathrm{mg}$ oral mifepristone administered by a health provider, $400 \mu \mathrm{g}$ oral misoprostol 48 hours later, and a follow-up visit after 14 days to confirm abortion outcome. Medical abortion has since become available in the private 


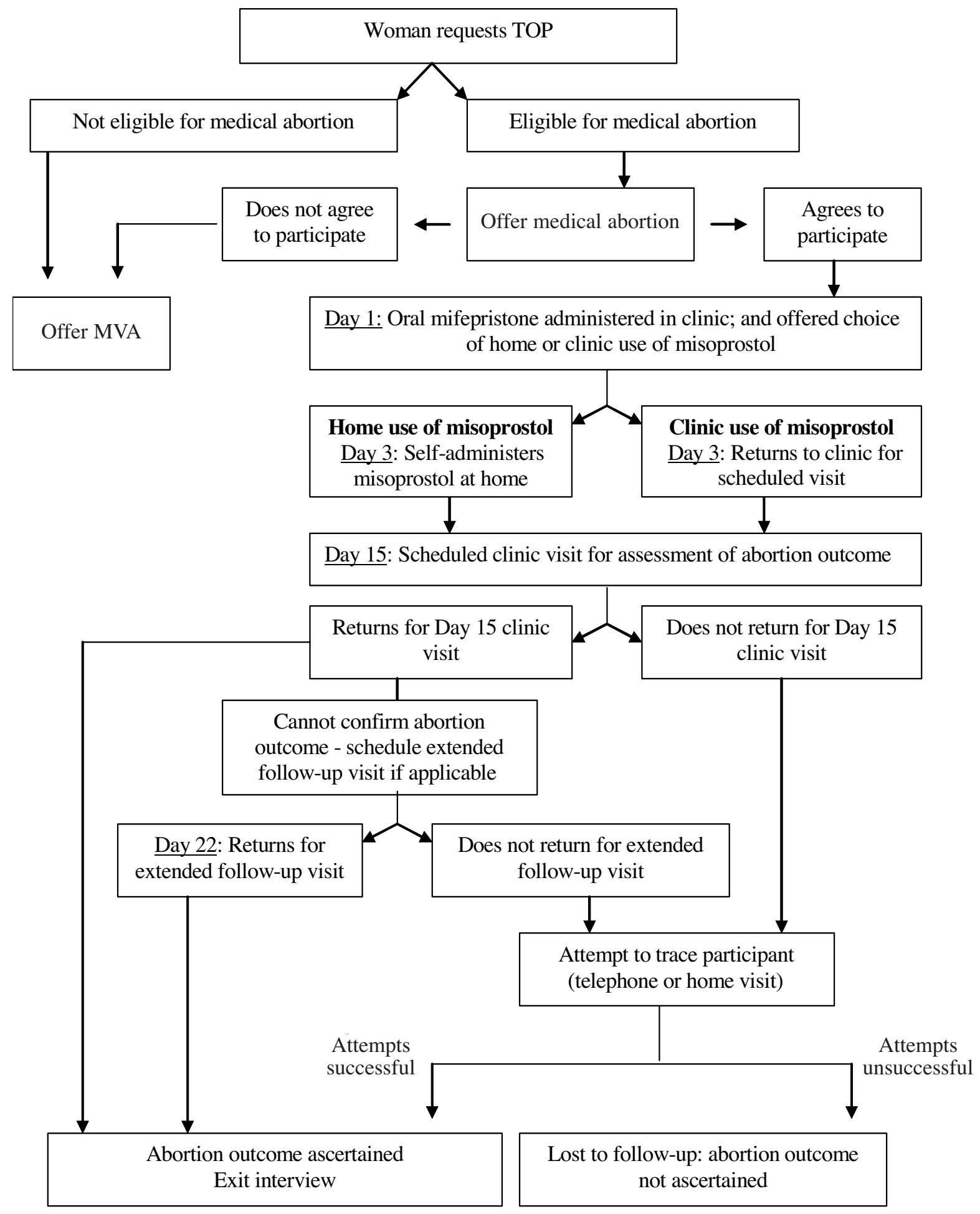

Figure 1 Participant recruitment and follow-up for the medical abortion regimen. MVA, manual vacuum aspiration; TOP, termination of pregnancy

health sector but has not yet been introduced in the public sector. Offering medical abortion in the public sector could complement existing MVA services in South Africa. It could potentially increase women's access to abortion, as it may require less staff input, and could be offered in facilities where MVA is not currently available and specialised equipment is not necessary.

The aim of this study was to evaluate the feasibility of integrating mifepristone-misoprostol medical abortion into existing abortion services in three pilot sites in South Africa, to inform the National Department of Health on how medical abortion could be integrated into current public health sector TOP services. To determine feasibility, the study evaluated operational requirements for providing medical abortion, women's and provider's acceptability of the method, pregnancy outcomes, interest in home use of misoprostol, and patient follow-up.

\section{Methods}

A prospective operations research study was conducted during the period January to May 2004. Three sites, located in two provinces in South Africa, were chosen for convenience; Site 1 is a secondary hospital in Cape Town, Site 2 a tertiary academic hospital in Tshwane, and Site 3 
consists of two primary care clinics in Johannesburg. All sites are existing TOP service points where trained midwives perform MVA for pregnancies up to 12 weeks. This was an exploratory study designed primarily to recruit sufficient numbers of participants to make a reasonable assessment of operational requirements for introducing medical abortion. A sample of at least 60 participants per health facility was deemed adequate for providers to gain sufficient experience of medical abortion for a reasonable assessment of operational requirements. This sample was thus not primarily selected to allow comparison of practices, experiences or outcomes between sites.

Five health providers (one in Site 1 and two each in Sites 2 and 3) provided medical abortion in the study. They all performed MVA, but had no experience with medical abortion. Before the study, these health providers and five other personnel from the sites attended a 2.5-day training workshop to orientate them to the medical abortion protocol. The workshop included theoretical information: the history and development of medical abortion; mechanisms of action and side effects of mifepristonemisoprostol regimens; epidemiology, global experience, and best practices for patient management; and practical training in providing medical abortion.

All women aged 18 years or older requesting TOP at less than 56 days from LMP during the study period were given the option of medical abortion or MVA to terminate the pregnancy. An eligibility checklist was administered to those who expressed interest in medical abortion and in the study. Inclusion criteria were: 18 years or older; live or work within 1 hour of a study site; gestational age of $\leq 56$ days; in general good health; and willing to provide an address and/or telephone number for follow-up. Exclusion criteria were: medical contraindication to mifepristonemisoprostol use and unwillingness to undergo a surgical abortion should the medical procedure fail. In all three sites pregnancy was confirmed with a conventional urine pregnancy test. All sites routinely used existing ultrasound facilities to assess gestational age for MVA. However, in this study ultrasound examination was not a prerequisite for confirmation of gestational age or abortion outcome. Providers' choice of method (ultrasound or clinical assessment) was on a case-by-case basis and depended on the norm in the facility.

Women who were interested in participating and were eligible signed a consent form and were included in the study. Figure 1 depicts the medical abortion regimen: $600 \mathrm{mg}$ oral mifepristone on Day 1, $400 \mu \mathrm{g}$ oral misoprostol 48 hours later, and a follow-up visit at the facility after 14 days to confirm abortion outcome. In all three sites mifepristone was administered in the health facility. Sites 1 and 3 offered home use of misoprostol, while Site 2 did not because hospital authorities disallowed off-site use of misoprostol. All sites were asked to trace women who did not return for a scheduled follow-up visit.
Abortion outcome was classified as: complete abortion (did not require surgical intervention), incomplete abortion or ongoing pregnancy (a viable fetus in utero).

Standardised instruments collected data on the experience of offering medical abortion, women's and providers' acceptability of the method, and experience and outcome of medical abortion. A study register documented the contact details of study participants, and the numbers of women who were eligible, had a medical TOP and returned for follow-up.

All participants completed a home study card to record side effects and medication use at home. Participants who returned on Day 15 had an exit interview to assess acceptability, and completed a standardised pain chart (pain was scored on a scale of 1 to 7 , where 1 represented no pain and 7 the worst pain). ${ }^{17,18}$ Exit interviews for women who did not return for a follow-up visit were done by telephone or at home visits. Qualitative interviews were conducted with five providers (three in Site 1 and two in Site 2) to assess their acceptability of medical abortion. Summary statistics were employed to analyse the data using the Statistical Package for the Social Sciences Version 12.0 (SPSS) (SPSS Inc., Chicago, IL, USA).

\section{Ethical approval}

The Committee for Research on Human Subjects (Medical) of the University of the Witwatersrand and the Research and Ethics Committee at the University of Cape Town approved the study protocol.

\section{Results}

A total of 290 women met the eligibility criteria and agreed to participate in the study. The participant profiles were similar in the three sites; the median age was 25 years, the majority of participants were single and had completed high school (Table 1). Only four participants reported having had a previous induced abortion.

Of the 290 women enrolled in the study, 29 (10\%) were lost to follow-up and $261(90 \%)$ had a confirmed abortion outcome, of whom $243(93.1 \%)$ had a complete abortion (Table 2). There were no statistically significant differences in median age, parity and education between women who completed the protocol and those lost to follow-up. All participants in Site 2 returned to the clinic for misoprostol; while in Sites 1 and 3, where women had a choice, the vast majority opted to take misoprostol at home (Site 1: $83.3 \%$; Site 3: 90.1\%).

Providers used more than one method to assess gestational age and abortion outcome. Overall, ultrasound was the most frequently used method for gestational age determination; both participant history and ultrasound were used to confirm outcome, except at Site 1 which did not use ultrasound at all to assess abortion outcome (Table 3).

The majority of the 261 women who had an exit interview (Table 4 ) were very satisfied with the experience $(96.4 \%)$ and would choose home use of misoprostol if they

Table 1 Profile of study participants

\begin{tabular}{llccc}
\hline Characteristic & $\begin{array}{l}\text { Cape Town } \\
(\boldsymbol{n}=\mathbf{6 0 )}\end{array}$ & $\begin{array}{l}\text { Tshwane } \\
(\boldsymbol{n}=\mathbf{1 1 0})\end{array}$ & $\begin{array}{l}\text { Johannesburg } \\
(\boldsymbol{n}=\mathbf{1 2 0})\end{array}$ & $\begin{array}{l}\text { All sites } \\
(\boldsymbol{n}=\mathbf{2 9 0})\end{array}$ \\
\hline Age (years) [median (IQR)] & $27.0(8.0)$ & $24.0(9.0)$ & $25.0(9.0)$ & $25.0(9.0)$ \\
Gravidity [mean (SD)] & $2.3(1.2)$ & $2.1(1.2)$ & $2.3(1.1)$ & $2.2(1.2)$ \\
Parity [mean (SD)] & $1.3(1.1)$ & $1.1(1.2)$ & $1.3(1.0)$ & $1.2(1.1)$ \\
Marital status & $(n=60)$ & $(n=106)$ & $(n=116)$ & $(n=282)$ \\
$\quad$ Married (\%) & 11.7 & $(n .7$ & 9.5 & 8.5 \\
Education & $(n=60)$ & $1.7=110)$ & $(n=119)$ & $(n=289)$ \\
$\quad$ Completed high school or higher (\%) & 95.0 & 100.0 & 96.6 & 97.6 \\
\hline
\end{tabular}

$\mathrm{IQR}$, interquartile range; SD, standard deviation. 
Table 2 Medical abortion outcome

\begin{tabular}{llllr}
\hline Outcome & Cape Town & Tshwane & Johannesburg & All sites \\
\hline Follow-up of women $[n(\%)]$ & $(n=60)$ & $(n=110)$ & $(n=120)$ & $(n=290)$ \\
Women with confirmed abortion outcome & $53(88.3)$ & $93(84.5)$ & $115(95.8)$ & $261(90.0)$ \\
Women lost to follow-up & $7(11.7)$ & $17(15.5)$ & $(4.2)$ & $29(10.0)$ \\
$\begin{array}{l}\text { (abortion outcome unconfirmed) } \\
\text { Abortion outcome* } n(\%)]\end{array}$ & $(n=53)$ & $(n=93)$ & $(n=115)$ & $(n=261)$ \\
$\begin{array}{l}\text { Success } \\
\quad \text { Complete abortion }\end{array}$ & $46(86.8)$ & $91(97.8)$ & $106(92.2)$ & $243(93.1)$ \\
$\begin{array}{l}\text { Failure } \\
\text { Incomplete abortion }\end{array} \quad 5(9.4)$ & $2(2.2)$ & $7(6.1)$ & $14(5.4)$ \\
$\quad$ Had an MVA for medical reason & $2(3.8)$ & $0(0.0)$ & $2(1.7)$ & $4(1.5)$ \\
\hline
\end{tabular}

*Excludes women who were lost to follow-up. MVA, manual vacuum aspiration.

Table 3 Methods used to determine gestational age and abortion outcome

\begin{tabular}{lllll}
\hline Method* $^{*}$ & Cape Town & Tshwane & Johannesburg & All sites \\
\hline To determine gestational age (\%) & $(n=60)$ & $(n=110)$ & $(n=120)$ & $(n=290)$ \\
Clinical examination & 11 & 100 & 3 & 44 \\
Ultrasound & 100 & 74 & 98 & 90 \\
To determine abortion outcome (\%) & $(n=53)$ & $(n=93)$ & $17=115)$ & $(n=261)$ \\
Clinical examination & 66 & 36 & 95 & 61 \\
Participant history & 98 & 90 & 98 & 75 \\
Ultrasound & 0 & & 95 & 75 \\
\hline
\end{tabular}

*Multiple response categories.

Table 4 Acceptability of medical abortion to women

\begin{tabular}{|c|c|c|c|c|}
\hline Reported acceptability & $\begin{array}{l}\text { Cape Town } \\
(n=60)\end{array}$ & $\begin{array}{l}\text { Tshwane } \\
(n=110)\end{array}$ & $\begin{array}{l}\text { Johannesburg } \\
(n=120)\end{array}$ & $\begin{array}{l}\text { All sites } \\
(n=290)\end{array}$ \\
\hline $\begin{array}{l}\text { Medical abortion was satisfactory or very } \\
\text { satisfactory }(\%)\end{array}$ & 96.0 & 97.8 & 95.5 & 96.4 \\
\hline $\begin{array}{l}\text { Was very prepared for what happened during } \\
\text { medical abortion (\%) }\end{array}$ & 83.7 & 97.8 & 91.1 & 92.1 \\
\hline $\begin{array}{l}\text { Medical abortion experience was not at all } \\
\text { difficult }(\%)\end{array}$ & 75.5 & 97.8 & 86.6 & 88.6 \\
\hline $\begin{array}{l}\text { Would choose home use of misoprostol if needed } \\
\text { another medical abortion (\%) }\end{array}$ & 89.9 & 89.9 & 99.1 & 94.0 \\
\hline \multicolumn{5}{|l|}{ Experience of pain } \\
\hline $\begin{array}{l}\text { Pain score [mean (SD)] } \\
\text { Took pain medication at home (\%) }\end{array}$ & $\begin{array}{l}4.5(2.0) \\
89.8\end{array}$ & 7.5 & $\begin{array}{l}4.4(1.8) \\
81.6\end{array}$ & $\begin{array}{l}3.9(1.8) \\
56.3\end{array}$ \\
\hline
\end{tabular}

SD, standard deviation.

ever needed another medical abortion (94\%). The absence of side effects, the quick and easy procedure, and knowing what to expect were reported as the best features of medical abortion, while pain and prolonged bleeding were the worst features for $23 \%$ and $16 \%$ of women, respectively.

No serious side effects were reported in this study. Pain, experienced by $66 \%$ of women who had an exit interview, and heavy bleeding $(67 \%)$ were the commonest side effects. The median duration of heavy bleeding and cramps were 2 days [interquartile range (IQR) 2] and 2 days (IQR 3), respectively, and $56 \%$ of the women took medication for pain (Table 4). During the study, 10 participants visited another health facility on account of prolonged bleeding. Seven had an MVA at the other facility, and one woman had an MVA and a blood transfusion [for a haemoglobin $(\mathrm{Hb})$ level of 7.4].

From their experience in this study, providers were of the opinion that introducing medical abortion could reduce their workload as it is an easier procedure to perform than MVA, and in addition it could decrease the number of MVAs performed. Providers were very keen to provide medical abortion in their facilities as it would allow earlier TOP and give women the opportunity to take misoprostol at home, thus requiring fewer visits to a health facility. Their concerns were that women might not return for follow-up visits, and a cut-off of 56 days from LMP may limit access to medical abortion for some women.

Providers identified prerequisites for integrating medical abortion in MVA services as follows: staff training; education of users about the availability of medical abortion, the 56 days from LMP cut-off, and compliance with follow-up schedule; and designated space for medical abortion. Only two of the five providers felt ultrasound should be a requirement. Providers reported that it should be possible for their facilities to continue providing medical abortion after the study, as long as barriers such as lack of space, insufficient privacy and the cost of medication are addressed. Their recommendation was for medical abortion services to be more extensively promoted, and for health authorities to encourage introducing medical abortion to complement MVA services.

\section{Discussion}

This study sought to highlight the operational requirements for introducing medical abortion in the public sector, and to determine the acceptability of medical abortion to women and health care providers. Results demonstrate that integration of medical abortion into existing public sector TOP services is achievable, and that medical abortion can 
be safely and successfully provided and is acceptable to women and health care providers.

The abortion success rate of $93 \%$ observed in this study is comparable to international experience for medical abortion at gestational age $\leq 56$ days from LMP. 5,6 Variation in success rates between sites could possibly be due to differences in provider comfort with medical abortion; some providers may have been less comfortable with the procedure and more likely to err on the side of caution when assessing outcome. The level and depth of counselling may be another factor, and relying on participant history may not be sufficient to assess abortion outcome in the absence of adequate counselling. Though all were experienced MVA providers, none had ever performed medical abortion. Greater experience with medical abortion may improve the success rates observed in this study.

Given the option, most women chose to take misoprostol at home, with successful outcomes. Similar experiences have been reported elsewhere; success rates of 92-97\% have been achieved with home use of misoprostol in the USA and several middle-income countries. ${ }^{13,14,19,20}$ Though not powered to evaluate differences between sites, our study suggests that abortion outcomes for home and clinic use of misoprostol are comparable.

Follow-up of women is essential to identify ongoing pregnancy, incomplete abortion or other complications of the procedure. In our study $10 \%$ of women were lost to follow-up, with Site 2 experiencing the greatest attrition. Variations could be due to differences in service provision: while Sites 1 and 3 proactively traced women with telephone calls and home visits, Site 2 did not do so on account of logistical barriers. Differential follow-up rates could also be attributed to client sensitivity: most women in Sites 1 and 3 took misoprostol at home and may have felt the need to return to the clinic for assurance of a complete abortion, while women in Site 2 who all returned to the clinic on Day 3 and received provider input at that stage may have felt less compelled to return for follow-up. However, since this study was not designed to evaluate differences across sites, we recommend further research to validate differences and identify their determinants. Nevertheless, the pattern observed here suggests that a twovisit medical abortion protocol including home use of misoprostol does not necessarily compromise patient follow-up.

A South African study conducted in a setting where only MVA was available and women were aware of the 12week cut-off found that $22 \%$ of all women requesting TOP came within the cut-off of 56 days from LMP, and so would be eligible for medical abortion if it were available in the public sector. ${ }^{21}$ This study dispels the myth that women do not seek TOP early enough to be eligible for medical abortion, and suggests that a greater proportion of women would probably request TOP before 56 days from LMP if medical abortion were available and deliberate efforts made to educate and inform them about the 56 days from LMP cut-off.

Acceptability of medical abortion among women is very high, and is similar for home and clinic use of misoprostol. $6,10,22$ When offered a choice between surgical and medical abortion, $60-70 \%$ of participants in clinical trials choose medical abortion, ${ }^{19}$ largely because of increased privacy, greater patient control over the procedure, and the option of avoiding instrumentation of the uterus. Similarly, medical abortion was highly acceptable for the vast majority of women in this study. Women in Site 2 had the greatest acceptability, recorded the lowest pain score, and took the least pain medication compared to their counterparts in Sites 1 and 3. Reasons for these differences are not immediately evident, but perhaps the additional provider input women in Site 2 received at the Day 3 visit may have played a role. However, sample sizes are too small to make valid comparisons; further research with larger samples is required to evaluate the determinants of client acceptability.

Serious side effects and other complications are rarely reported with the mifepristone-misoprostol abortion regimen. 1,6,22 Side effects experienced in this study are consistent with other studies internationally: bleeding and pain, the most common and consistently reported effects in this study, occurred for both home use and clinic use of misoprostol. In our study, seven of ten women who visited facilities outside the study sites due to prolonged bleeding had an MVA at the other facility, suggesting a lack of awareness and knowledge about medical abortion amongst clinicians in the health sector. A case in point is the woman with an $\mathrm{Hb}$ level of 7.4 who received a blood transfusion for prolonged bleeding. Notwithstanding the absence of clinical information other than her $\mathrm{Hb}$ level, it would seem that an MVA alone would have sufficed to manage this woman. The lesson learnt is that MVA should be available as a backup for the small proportion of medical abortion clients who will need surgical intervention for ongoing pregnancy, incomplete abortion or excessive bleeding. However, training of providers (including those who do not routinely perform medical abortion) on the medical abortion procedure, clinical management guidelines and indications for intervention are essential to avoid unnecessary interventions. This may require a widespread education campaign targeting all health providers, coupled with in-depth client counselling programmes to explain the high likelihood of bleeding and cramps in the medical abortion process, and to advise on appropriate actions should clients experience these for prolonged periods.

Our provider interviews, though only applicable to Sites 1 and 2 and not nationally representative, probably reflect views of urban TOP providers. These encouragingly suggest that medical abortion is acceptable to health care providers who are already providing MVA. This resonates with experience elsewhere, suggesting that medical abortion may be acceptable to providers who are already engaged in providing surgical abortion. ${ }^{23}$ Notably, providers in our study felt that there may be wider acceptability of a TOP method that they themselves did not have to initiate.

Ultrasound was frequently used in this study probably because it was readily available, and providers were perhaps unduly cautious because of their limited experience with medical abortion. However, experience shows that ultrasound is not mandatory for effective provision of medical abortion, and that providers' clinical assessment of gestational age correlates closely to ultrasound assessment.1,21 A South African study showed that midwives can accurately date pregnancies of less than 56 days from LMP by clinical assessment. ${ }^{21}$ With appropriate training and experience providers should rely less on ultrasound and more on clinical assessment.

This study used the MCC-approved medical abortion protocol of $600 \mathrm{mg}$ mifepristone, but a less costly $200 \mathrm{mg}$ mifepristone regimen is just as effective. ${ }^{4,11}$ Moreover, drug costs need not be a deterrent to introducing medical abortion as the cost of a nurse providing a first-trimester MVA at primary care level in the South African public sector is similar to the cost of providing a medical abortion with $200 \mathrm{mg}$ mifepristone. ${ }^{24,25}$ Further cost savings could be achieved with a two-visit medical abortion protocol allowing home use of misoprostol. 


\section{Conclusions}

This operations research study - the first use of medical abortion in the public sector in South Africa - shows that integration of medical abortion into the South African public health care sector is feasible, and that medical abortion can be safely and successfully provided and is acceptable to women and health care providers. An enabling legislative framework already exists for the integration of medical abortion into public sector TOP services. In the context of sexual and reproductive rights, integrating medical abortion would expand the choice of abortion methods available to women, and increase TOP access for women seeking an early TOP in South Africa.

\section{Acknowledgements}

The authors would like to acknowledge the women and health care providers who participated in this study, and the management of the health facilities. They would also like to thank their colleagues at the National Abortion Federation (USA) and the Centre for Training in Reproductive Health Technologies (France) who conducted the medical abortion training for the health providers in this study.

\section{Statements on funding and competing interests}

Funding Financial support for this study was provided by the MediTeam Trust (Johannesburg, South Africa) and Gynuity Health Projects (New York, NY, USA).

Competing interests None identified.

\section{References}

1 Kahn JG, Becker BJ, Maclsaa L, Amory JK, Neuhaus J, Olkin I, et al. The efficacy of medical abortion: a meta-analysis. Contraception 2000; 61: 29-40.

2 Peyron R, Aubény E, Targosz V, Silvestre L, Renault M, Elkik F, et al. Early termination of pregnancy with mifepristone (RU 486) and the orally active prostaglandin misoprostol. $N$ Engl J Med 1993; 328: 1509-1513.

3 El-Rafaey H, Rajasekar D, Abdalla M, Calder L, Templeton A Induction of abortion with mifepristone (RU 486) and oral or vaginal misoprostol. N Engl J Med 1995; 332: 983-987.

4 Grimes D. Medical abortion in early pregnancy: a review of the evidence. Obstet Gynecol 1997; 89: 790-796.

5 Spitz IM, Bardin CW, Benton L, Robbins A. Early pregnancy termination with mifepristone and misoprostol in the United States. N Engl J Med 1998; 338: 1241-1247.

6 Winikoff B, Sivin I, Coyaji KJ, Cabezas E, Xiao B, Gu S, et al. Safety, efficacy, and acceptability of medical abortion in China, Cuba and India: a comparative trial of mifepristone-misoprostol versus surgical abortion. Am J Obstet Gynecol 1997; 176: 431-437.

7 Coyaji K, Elul B, Krishna U, Otiv S, Ambardekar S, Bopardikar $\mathrm{A}$, et al. Mifepristone abortion outside the urban research hospital setting in India. Lancet 2001; 357(9520): 120-122.

8 Henderson JT, Hwang AC, Harper CC, Stewart FH. Safety of mifepristone abortions in clinical use. Contraception 2005; 72 : 175-178.

9 Jones RK, Henshaw SK. Mifepristone for early medical abortion: experiences in France, Great Britain and Sweden. Perspect Sex Reprod Health 2002; 34: 154-161.

10 Elul B, Hajri S, Ngoc NN, Ellerston C, Slama CB, Pearlman E, et al. Can women in less-developed countries use a simplified medical abortion regimen? Lancet 2001; 357(9266): 1402-1405.

11 World Health Organization Task Force on Post-Ovulatory Methods of Fertility Regulation. Comparison of two doses of mifepristone in combination with misoprostol for early medical abortion: a randomised trial. Br J Obstet Gynaecol 2000; 107: 524-530.

12 Schaff EA, Eisinger SH, Stadalius LS, Franks P, Gore BZ, Poppema S. Low-dose mifepristone $200 \mathrm{mg}$ and vaginal misoprostol for abortion. Contraception 1999; 59: 1-6.

13 Akin A, Blum J, Ozalp S, Ondero lu L, Kirca U, Bilgili N, et al. Results and lessons learned from a small medical abortion clinical study in Turkey. Contraception 2004; 70: 401-406.

14 Hajri S, Blum J, Gueddana N, Saadi H, Maazoun L, Chélli H, et al. Expanding medical abortion in Tunisia: women's experiences from a multi-site expansion study. Contraception 2004; 70: 487-491.

15 Department of Health. Choice on Termination of Pregnancy Act, 1996 (Act 92 of 1996). Pretoria, South Africa: Department of Health, 1996.
16 Department of Health. Termination of Pregnancy Implementation Guidelines. Pretoria, South Africa: National Directorate of Maternal, Child and Women's Health (not dated).

17 Blanchard K, Shochet T, Coyaji K, Ngoc NTN, Winikoff B. Misoprostol alone for early abortion: an evaluation of seven potential regimens. Contraception 2005; 72: 91-97.

18 Blanchard K, Taneepanichskul S, Kiriwat O, Sirimai K, Svirirojana N, Mavimbela N, et al. Two regimens of misoprostol for treatment of incomplete abortion. Obstet Gynecol 2004; 103: 860-865.

19 Winikoff B. Acceptability of medical abortion in early pregnancy. Fam Plann Perspect 1995; 27: 142-148.

20 Akin A, Dabash R, Dilbaz B, Dursun P, Aktun H, Gokkurt S, et al. Increasing women's choice and access to safe abortion services in Turkey: a study of the efficacy and acceptability of medical abortion in four sites. Reproductive Health 2005 Meeting Abstracts, 7-10 September 2005, St Petersburg, FL, USA. Contraception 2005; 72: 237-238.

21 Cooper D, Dickson K, Blanchard K, Cullingworth L, Brown $\mathrm{H}$, Mavimbela $\mathrm{N}$, et al. Medical abortion: the possibilities for introduction in the public sector in South Africa. Reprod Health Matters 2005; 13: 35-43.

22 Ngoc NT, Winikoff B, Clark S, Ellertson C, Am KN, Do TH, et al. Safety, efficacy and acceptability of mifepristone-misoprostol abortion in Vietnam. Int Fam Plan Perspect 1999; 25: 10-14.

23 Joffe C. Reactions to medical abortion among providers of surgical abortion: an early snapshot. Fam Plann Perspect 1999; 31: 39-43.

24 de Pinho H. Cost Analysis of Abortion Performed in the Public Sector. Cape Town, South Africa: Women's Health Research Unit, Department of Public Health, University of Cape Town, 2000.

25 Cullingworth L, de Pinho H. A Cost Analysis of Service Provision of Medical Abortions in the Public Health Sector at Primary and Secondary Level. Cape Town, South Africa: Women's Health Research Unit, Department of Public Health, University of Cape Town, 2002

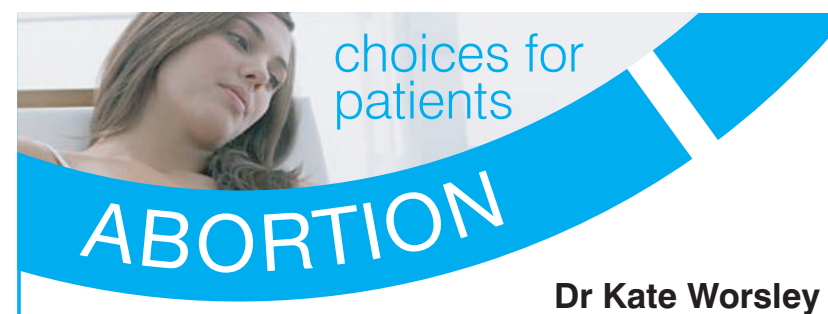

Head of Medical Development - Marie Stopes International

Many women who fall pregnant unexpectedly do not wish to continue with their pregnancy and it is important to provide them with choice, support and professional abortion services to help them get their lives back on track.

Marie Stopes International now sees one third of all women seeking abortion in England and Wales. As experts in this field they have pioneered and modernised abortion provision making them first choice amongst healthcare professionals.

Medical abortion - currently one third of women between 4-9 weeks gestation having abortion, choose the abortion pill. At Marie Stopes centres this has been simplified to 2 visits over 2 days.

Surgical abortion - women prefer a quick, convenient appointment and the majority are now choosing Marie Stopes one visit only appointments where the consultation and treatment are provided on the same day. A choice of anaesthesia including local, conscious sedation and general anaesthetic is also offered by the organisation.

A 24 hour appointment booking line and aftercare service on 08451203644 offers all the support and advice clients need.

Call for a Marie Stopes International GP Sexual Health Pack containing information on current abortion treatment choices and referral guidelines.

08451203644 www.mariestopes.org.uk working with you...

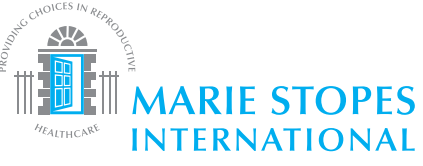

CONTRACEPTION • HEALTH SCREENING • STERILISATION • VASECTOMY • ABORTION 